\title{
Спектроскопия комбинационного рассеяния, инфракрасного поглощения и люминесценции нитрида алюминия, легированного бериллием
}

\author{
(С) И.Д. Бреев ${ }^{1}$, В.Д. Яковлева ${ }^{1}$, О.С. Кудрявцев ${ }^{2}$, П.Г. Баранов ${ }^{1}$, Е.Н. Мохов ${ }^{1}$, А.Н. Анисимов ${ }^{1, \text { ฯ }}$ \\ ${ }^{1}$ Физико-технический институт им. А.Ф. Иоффре Российской академии наук, \\ 194021 Санкт-Петербург, Россия \\ ${ }^{2}$ Институт общей фризики им. А.М. Прохорова Российской академии наук, \\ 119991 Москва, Россия \\ ฯE-mail: aan0100@gmail.com
}

Поступила в Редакцию 12 ноября 2020 г.

В окончательной редакции 23 ноября 2020 г.

Принята к публикации 23 ноября 2020 г.

Исследовано влияние высокотемпературной $\left(T=1880^{\circ} \mathrm{C}\right)$ диффузии ионов бериллия и электронного облучения на оптические свойства монокристаллического нитрида алюминия. Показано, что введение Ве в AlN приводит к изменению спектральных характеристик комбинационного рассеяния света и инфракрасного поглощения. Анализ спектров комбинационного рассеяния света и инфракрасного поглощения в кристаллах, содержащих примесь Ве, доказывает, что эта примесь является геттером собственных дефектов, ответственных за желтый цвет кристалла AlN и уширение линий в спектрах.

Ключевые слова: нитрид алюминия, легирование, бериллий, геттер, собственные дефекты, комбинационное рассеяние света, фотолюминесценция, ифракрасное поглощение.

DOI: 10.21883/FTP.2021.03.50603.9554

\section{1. Введение}

Нитрид алюминия (AlN) является полупроводником с экстремально широкой запрещенной зоной $\left(E_{g}=6.1\right.$ эВ) [1]. Это свойство, в совокупности с уже развитой технологией получения объемных монокристаллов AlN [2], позволяет получать на подложках AlN светодиодные структуры на основе тринитридов (InGaN, AlGaN) [3]. Относительно недавно уже продемонстрировано излучение в ультрафиолетовом (УФ) диапазоне (210 нм) $p-i-n$-диодных структур на основе $\mathrm{AlN}$, легированных $\mathrm{Si}$-донор и $\mathrm{Mg}$-акцептор [4]. Для повышения эффективности данных структур, во-первых, необходимо минимизировать оптическое УФ поглощение в подложках AlN. Bo-вторых, чтобы сделать такие устройства более эффективными, требуются исследования влияния донорных и акцепторных примесей. Донорные примеси ( $\mathrm{Si}$ и $\mathrm{O}$ ) изучены довольно полно [5-7], тогда как акцепторные примеси второй группы, такие как $\mathrm{Be}$ и $\mathrm{Li}$, мало изучены. Тем не менее недавнее исследования монокристаллического AlN, легированного Be посредством высокотемпературной диффузии из паровой фазы, показали, что Ве формирует глубокие уровни акцепторного типа $[8,9]$. Это может быть использовано для компенсации неконтролируемых примесных центров $n$-типа, легирующих AlN в ходе роста, таких как кислород и кремний [7], что необходимо для получения монокристаллов AlN c экстремально высоким удельным сопротивлением.

\section{2. Технология приготовления кристаллов и методики исследования}

Исследовались кристаллы AlN, выращенные методом сублимации на затравках AlN. Условия роста и используемые ростовые установки описаны ранее $[10,11]$. После выращивания кристаллы разрезались на пластины толщиной 0.5 мм, поверхность которых полировалась. Основными легирующими примесями были кислород, углерод и кремний, содержание которых, определенное вторичной ионной масс-спектроскопией (ВИМС), составляло: $\mathrm{N}(\mathrm{O})=5.6 \cdot 10^{18}, \quad \mathrm{~N}(\mathrm{C})=4.5 \cdot 10^{18}$ и $\mathrm{N}(\mathrm{Si})=4.8 \cdot 10^{17} \mathrm{~cm}^{-3} \quad$ соответственно. Кристаллы имели янтарную или желтую окраску, обусловленную присутствием кислорода [12] или ассоциатов, включающих вакансию алюминия $\left(V_{\mathrm{Al}}\right)$ [13]. Далее в пластины AlN путем высокотемпературной диффузии вводилась примесь бериллия. Диффузия осуществлялась в замкнутых тиглях из карбида тантала (ТaC) при температуре $1600-1800^{\circ} \mathrm{C}$. Продолжительность отжига составляла 2 ч. При этих условиях Ве диффундировал на всю глубину кристалла, что контролировалось по изменению спектра люминесценции и цвета образца.

\section{3. Экспериментальные результаты и обсуждение}

Были проанализированы 5 образцов, изготовленных из одной пластины AIN. Из них 3 образца содержали $\mathrm{Be}$, введенный путем диффузии, один из которых был 
облучен электронами. Два образца, не содержащие примеси Ве, были использованы в качестве эталонов, один из них также был облучен электронами.

\section{1. Спектроскопия комбинационного рассеяния света}

Образцы AlN были исследованы методом комбинационного рассеяния света (КРС) в области $180-1500 \mathrm{~cm}^{-1}$. Экспериментальные результаты представлены на рис. 1. Спектры образцов \#2-5, вырезанных в плоскости $n$ (0001), были получены в геометрии рассеяния $Z(X ;-)-Z$, а спектр образца \#1 вырезан по плоскости $m(11-20)$ в геометрии рассеяния $Y(Z ;-)-Y$. Для образцов \#2-5 отмечены пики, соответствующие фононным модам $\mathrm{E}_{2}$ (low), $\mathrm{E}_{2}$ (high), $\mathrm{E}_{1} \mathrm{TO}$ и $\mathrm{A}_{1}$ LO. Для образца \#1 появляются пики, связанные с фононной модой $\mathrm{A}_{1} \mathrm{TO}$ и квазипродольной оптической модой (QLO), которую описывают как приближение к $\mathrm{E}_{1}$ LO [14], а также лучше разрешается пик $\mathrm{E}_{1} \mathrm{TO}[15,16]$.

Для пиков, соответствующих фононной моде $\mathrm{E}_{2}$ (high), всех образцов проведена аппроксимация функцией Фогта и оценено значение полуширины линии, которые представлены в табл. 1. Можно видеть, что при легировании Ве и облучении электронами происходит уширение пика КРС. При легировании Ве кристалла AlN увеличение полуширины составляет $0.5 \mathrm{~cm}^{-1}$, при облучении электронами исходного $\mathrm{AlN}-3.6 \mathrm{~cm}^{-1}$, при облучении электронами $\mathrm{AlN}$, легированного $\mathrm{Be},-$ $0.5 \mathrm{~cm}^{-1}$. Уширение пиков КРС мы связываем с потерей кристаллического качества. Можно сделать вывод, что легированный Be кристалл $\mathrm{AlN}$ медленней теряет кристаллическое качество при облучении. Таким образом Ве является геттером радиационных дефектов, что приводит к минимизации нарушений в решетке, по сравнению с кристаллами, не содержащими примесь, что в свою очередь повышает радиационную стойкость AlN.

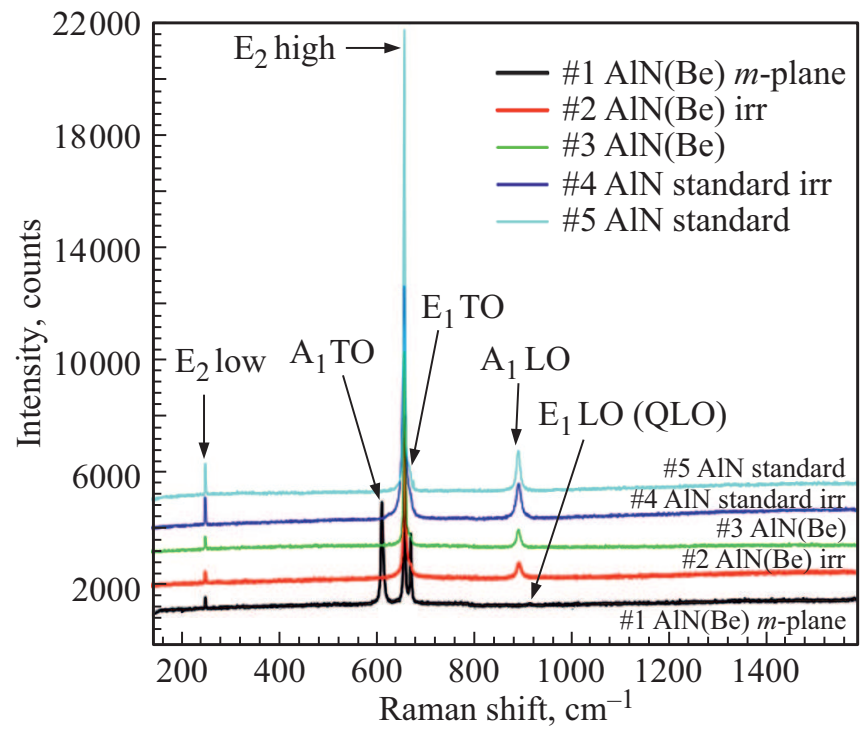

Рис. 1. Спектры КРС образцов AlN с отмеченными фононными модами.
Таблица 1. Анализ пиков КРC образцов AlN фононной моды $\mathrm{E}_{2}$ (высокий)

\begin{tabular}{l|c|c}
\hline \multicolumn{1}{c|}{ Образец } & $\begin{array}{c}\text { Амплитуда Е2 } \\
\text { (высокий), } \\
\text { см}^{-1} \cdot \text { счетов }\end{array}$ & $\begin{array}{l}\text { Полуширина Е } \\
\text { (высокий), см }\end{array}$ \\
\hline \#1 AlN(Be), m-плоскость & 30699.4851 & 3.30787 \\
\#2 AlN(Be), облученный & 37166.4302 & 4.2106 \\
\#3 AlN(Be) & 36706.346 & 3.71465 \\
\#4 AlN, стандартное & 88100.0163 & 6.83195 \\
облучение & 76187.4861 & 3.22504 \\
\#5 AlN, стандартный &
\end{tabular}

Таблица 2. Энергия фононных мод AlN [16]

\begin{tabular}{c|c|c}
\hline Мода & Энергия, мэВ & Энергия, см ${ }^{-1}$ \\
\hline $\mathrm{LO}$ & 91.4 & 735.3 \\
$\mathrm{TO}_{1}$ & 82.5 & 663.7 \\
$\mathrm{TO}_{2}$ & 78.1 & 628.3 \\
$\mathrm{LA}$ & 62.9 & 506 \\
$\mathrm{TA}_{1}$ & 55.3 & 444.9 \\
$\mathrm{TA}_{2}$ & 50.9 & 409.5
\end{tabular}

\section{2. Спектроскопия инфракрасного поглощения}

Было проведено исследование серии из 5 образцов $\mathrm{AlN}$ методом спектроскопии инфракрасного поглощения света с использованием ИК фурье-спектрометра Perkin Elmer Spectrum 100. Поглощение наблюдалось в диапазоне 950-3200 $\mathrm{cm}^{-1}$. Время экспозиции каждого спектра составило 20 мин.

По экспериментальным результатам был измерен коэффициент поглощения, нормированный на толщину образца. Для перевода единиц измерения использовалось соотношение

$$
T=\left(1-R^{\alpha}\right)^{\alpha} e^{-\alpha d} \quad \text { и } \quad R=\frac{(n-1)^{\alpha}}{(n+1)^{\alpha}},
$$

где $T$ - коэффициент пропускания, $R-$ коэффициент отражения, $n-$ показатель преломления, $\alpha-$ коэффициент поглощения, $d$ - толщина образца.

На рис. 2 приведены спектры инфракрасного поглощения 5 образцов серии AIN в области 950-1500 $\mathrm{cm}^{-1}$. Сплошной вертикальной линией со стрелочкой отмечено табличное значение, соответствующее 2 фононным комбинациям определенных фононных мод.

Энергия соответствующих фононных мод представлена в табл. 2 и указана на рис. 2. В данной работе мы не ставим цель подробно проводить анализ данных мод и приводим экспериментальные данные, дополнительно подтверждающие возможности метода ИК-спектроскопии. Наблюдаемые моды достаточно подробно описаны в статье [17].

На рис. 3 изображены спектры инфракрасного поглощения 5 образцов AlN в области $1550-2200 \mathrm{~cm}^{-1}$. 


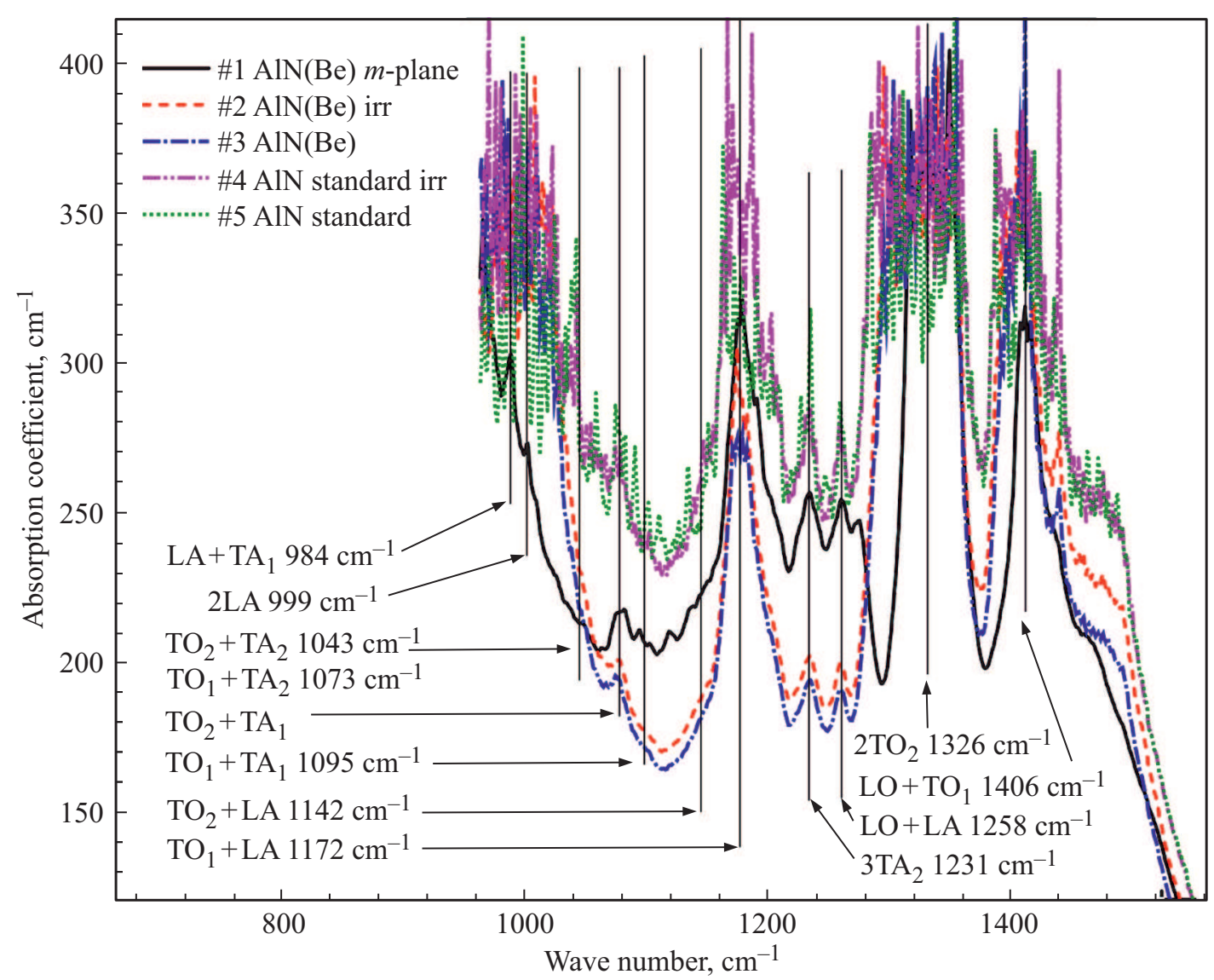

Pис. 2. Спектры инфракрасного поглощения 5 образцов AlN в области $950-1500 \mathrm{~cm}^{-1}$.

Разными линиями изображены спектры разных образцов серии, как указано в легенде сверху. На спектрах отмечены наблюдаемые линии, положение их пиков, а также

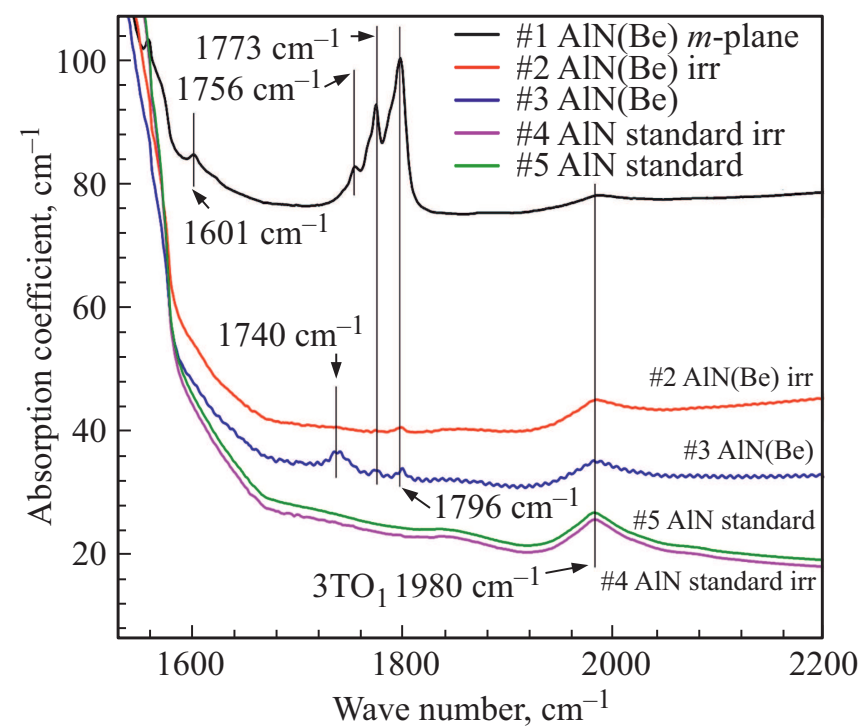

Рис. 3. Спектры инфракрасного поглощения 5 образцов AlN в области $1550-2200 \mathrm{~cm}^{-1}$. Разными линиями изображены спектры разных образцов, как указано в легенде сверху. На спектрах отмечены наблюдаемые линии и положение их пиков. предполагаемая природа их происхождения. Все линии можно разделить на 2 группы: линия $1980 \mathrm{~cm}^{-1}$ и линии в области $1700-1800 \mathrm{~cm}^{-1}$. Линия $1980 \mathrm{~cm}^{-1}$, согласно статьям [18], относится к поглощению в 3-фононном процессе с фононной модой $\mathrm{TO}_{1}$. Наибольший интерес в нашем исследовании представляют линии в диапазоне $1700-1800 \mathrm{~cm}^{-1}$. Они проявляются только при легировании Be кристаллов AIN, причем наиболее отчетливо видны для вырезанного в плоскости $m(11-20)$ образца $\mathrm{AlN}$, легированного Ве. Ранее в литературе линии в этом диапазоне наблюдались для трехосных центров С, но присутствовала только одна линия $1769 \mathrm{~cm}^{-1}$ для естественного изотопа $\mathrm{C}^{12}$ [17], что не совпадает с наблюдаемыми нами значениями в $1740 \mathrm{~cm}^{-1}$ и $1796 \mathrm{~cm}^{-1}$. Таким образом, предположительно, эти линии могут относиться к поглощению центров, введенных при диффузии Ве.

\section{3. Фотолюминесцентная спектроскопия}

Для 5 образцов AlN были проведены исследования фотолюминесценции (ФЛ) под ультрафиолетовым возбуждением светодиодной лампой M365LP1-C1 (Thorlabs) и сбором света с использованием конфокального оптического микроскопа Spectra (NT-MDT SI). Полученные спектры изображены на рис. 4. 


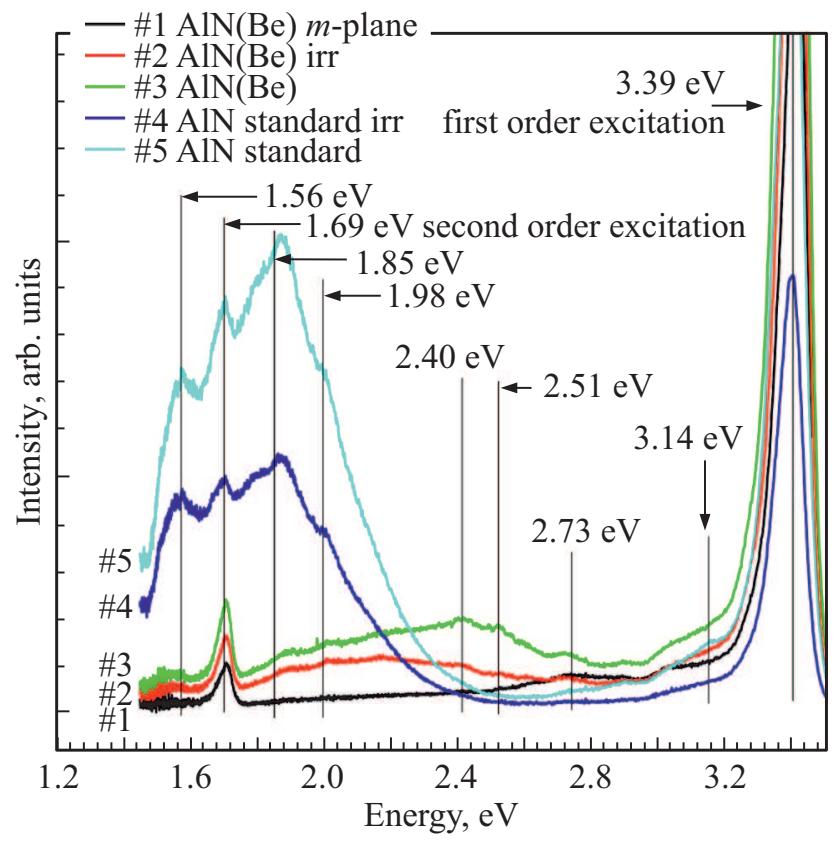

Рис. 4. Спектры фотолюминесценции 5 образцов $\mathrm{AlN}$ c возбуждением 3.39 эВ. Разными линиями изображены спектры разных образцов, как указано в легенде сверху.

На всех спектрах присутствует линия возбуждения ультрафиолетовой лампой 3.39 эВ и линия 2-го порядка возбуждения 1.69 эВ, являющаяся продуктом дифракции на решетке. На спектрах образцов AlN \#4 и \#5 видны линии $1.56,1.85$ и 1.98 эВ, которые подробно рассматриваются в работе [19]. Линию 1.56 эВ относят к оптическому переходу между валентной зоной и комплексом $\left(V_{\mathrm{Al}}-2 \mathrm{O}_{\mathrm{N}}\right)^{0}$. Линия 1.85 эВ соответствует оптическому переходу между валентной зоной и комплексом $\left(V_{\mathrm{Al}}-\mathrm{O}_{\mathrm{N}_{-} \mathrm{b}}\right)^{1-}$ в базальной конфигурации с $\mathrm{O}_{\mathrm{N}}$, расположенным в одной из трех тетраэдрических позиций. Линия 1.98 эВ относится к оптическому переходу между валентной зоной и комплексом $\left(V_{\mathrm{Al}}-\mathrm{O}_{\mathrm{N}_{-} \mathrm{a}}\right)^{1-}$ в аксиальной конфигурации, с $\mathrm{O}_{\mathrm{N}}$, расположенным вдоль оси с. Линия 3.14 эВ рядом с линией возбуждения может являться частью линии оптического перехода $\left(V_{\mathrm{Al}}-\mathrm{O}_{\mathrm{N}_{-} \mathrm{a}}\right)^{2-}$ - зона проводимости. Линии $2.40,2.51$ и 2.73 эВ появляются при легировании Ве образцов $\mathrm{AlN}$, при этом линии 2.51 и 2.73 эВ относят соответственно к переходам валентная зона $-V_{\mathrm{Al}}^{2-}$ и $V_{\mathrm{Al}}^{3-}-$ зона проводимости. Появление этих линий может свидетельствовать об увеличении концентрации собственных дефектов, таких как $V_{\mathrm{Al}}$. При этом образцы AlN, легированные $\mathrm{Be}$, имеют намного менее интенсивную люминесценцию линий $1.56,1.85$ и 1.98 эВ, что может свидетельствовать о разрушении комплексов $V_{\mathrm{Al}}-\mathrm{O}_{\mathrm{N}}$.

\section{4. Заключение}

Исследовано влияние высокотемпературной $\left(T=1880^{\circ} \mathrm{C}\right)$ диффузии ионов бериллия и электронного облучения на оптические свойства монокристаллического нитрида алюминия. Показано, что введение Be в AlN приводит к изменению спектральных характеристик комбинационного рассеяния света (КРС) и инфракрасного поглощения (ИП). Анализ спектров КРС и ИП в кристаллах, облученных быстрыми электронами, содержащих примесь Ве, доказывает, что эта примесь является геттером радиационных дефектов, что приводит к минимизации нарушений в решетке по сравнению с кристаллами, не содержащими примесь, что регистрируется по отсутствию уширения линий, наблюдаемому в КРС спектрах. Этот эффект обусловливает более высокую устойчивость материала к радиационному излучению. Существенное уменьшение интенсивности желтой окраски, наблюдаемой при введении Ве, очевидно, связано с захватом собственных дефектов ростового происхождения или примесных атомов, в частности кислорода, с образованием прочных стабильных ассоциатов с атомами бериллия.

\section{Финансирование работы}

Исследование выполнено при поддерже гранта Российского фонда фундаментальных исследований № 19-02-00649.

\section{Конфликт интересов}

Авторы заявляют, что у них нет конфликта интересов.

\section{Список литературы}

[1] J.Y. Tsao, S. Chowdhury, M.A. Hollis, D. Jena, N.M. Johnson, K.A. Jones, R.J. Kaplar, S. Rajan, C.G. van de Walle, E. Bellotti, C.L. Chua, R. Collazo, M. E. Coltrin, J.A. Cooper, K.R. Evans, S. Graham, T.A. Grotjohn, E.R. Heller, M. Higashiwaki, M.S. Islam, P.W. Juodawlkis, M.A. Khan, A.D. Koehler, J.H. Leach, U.K. Mishra, R.J. Nemanich, R.C.N. Pilawa-Podgurski, J.B. Shealy, Z. Sitar, M.J. Tadjer, A.F. Witulski, M. Wraback, J.A. Simmons. Adv. Electron. Mater., 4, 1600501 (2018).

[2] T.Y. Chemekova, O.V. Avdeev, I.S. Barash, E.N. Mokhov, S.S. Nagalyuk, A.D. Roenkov, A.S. Segal, Y.N. Makarov, M.G. Ramm, S. Davis, G. Huminic, H. Helava. Phys. Status Solidi C, 5, 1612 (2008).

[3] B. Monemar, P.P. Paskov, J.P. Bergman, A.A. Toropov, T.V. Shubina. Phys. Status Solidi B, 244, 1759 (2007).

[4] Y. Taniyasu, M. Kasu, T. Makimoto. Nature (London, UK), 441, 325 (2006).

[5] S.B. Orlinskii, J. Schmidt, P.G. Baranov, M. Bickermann, B.M. Epelbaum, A. Winnacker. Phys. Rev. Lett., 100, 256404 (2008).

[6] X.Th. Trinh, D. Nilsson, I.G. Ivanov, E. Janzn, A. Kakanakova-Georgieva, N.T. Son. Appl. Phys. Lett., 105, 162106 (2014).

[7] V.A. Soltamov, I.V. Ilyin, A.A. Soltamova, D.O. Tolmachev, N.G. Romanov, A.S. Gurin, V.A. Khramtsov, E.N. Mokhov, Yu.N. Makarov, G.V. Mamin, S.B. Orlinskii. Appl. Magn. Reson., 44, 1139 (2013). 
[8] V.A. Soltamov, M.K. Rabchinskii, B.V. Yavkin, O.P. Kazarova, S.S. Nagalyuk, V.Y. Davydov, A.N. Smirnov, V.F. Lebedev, E.N. Mokhov, S.B. Orlinskii, P.G. Baranov. Appl. Phys. Lett., 113, 082104 (2018).

[9] E.N. Mokhov, M.K. Rabchinskiy, S.S. Nagalyuk, M.R. Gafurov, O.P. Kazarova. Semiconductors, 54 (3), 278 (2020).

[10] E.N. Mokhov, A.A. Wolfson. Growth of AlN and GaN crystals by sublimation. In book: Single Crystals of Electronic Materials: Growth and Properties (Editted by Roberto Fornari, 2018) p. 401.

[11] O.V. Avdeev, T.Yu. Chemekova, E.N. Mokhov, S.S. Nagalyuk, H. Helava, M.G. Ramm, A.S. Segal, A.I. Zhmakin, Yu.N. Makarov. Development of 2"AlN Substrates Using SiC Seeds. Modern Aspects of Bulk Crystal and Thin Film Preparation (2012) p. 213-262.

[12] M. Tanaka, S. Nakahata, K. Sogabe, H. Nakata, M. Tobioka. J. Appl. Phys., 36, L1062 (1997).

[13] A. Sedhain, L. Du, J.H. Edgar, J.Y. Lin, H.X. Jiang. App. Phys. Lett., 95, 262104 (2009).

[14] F. Demangeot, J. Groenen, J. Frandon, M.A. Renucci, O. Briot, S. Clur, R.L. Aulombard. Appl. Phys. Lett., 72, 2674 (1998).

[15] V.Yu. Davydov, Yu.E. Kitaev, I.N. Goncharuk, A.N. Smirnov, J. Graul, O. Semchinova, D. Uffmann, M.B. Smirnov, A.P. Mirgorodsky, R.A. Evarestov. Phys. Rev. B, 58, 12899 (1998).

[16] M. Kuball, J.M. Hayes, Ying Shi, J.H. Edgar, A.D. Prins, N.W.A. van Uden, D.J.Dunstan. J. Cryst. Growth, 231, 391 (2001).

[17] A.T. Collins, E.C. Lightowlers, P.J. Dean. Phys. Rev., 158, 833 (1967).

[18] K. Irmscher, C. Hartmann, C. Guguschev, M. Pietsch, J. Wollweber, M. Bickermann. J. Appl. Phys., 114, 123505 (2013).

[19] Qin Zhou, Zhaofu Zhang, Hui Li, Sergii Golovynskyi, Xi Tang, Honglei Wu, Jiannong Wang, Baikui Li. APL Mater., 8, 081107 (2020).

Редактор Г.А. Оганесян

\section{Raman scattering, infrared absorption and photoluminescence spectroscopy of AIN doped by Be}

I.D. Breev ${ }^{1}$, V.D. Yakovleva ${ }^{1}$, O.S. Kudryavtsev' ${ }^{2}$, P.G. Baranov' ${ }^{1}$, E.N. Mokhov' ${ }^{1}$, A.N. Anisimov ${ }^{1}$

${ }^{1}$ loffe Institute,

194021 St. Petersburg, Russia

${ }^{2}$ Prokhorov General Physics Institute

of the Russian Academy of Sciences, 119991 Moskow, Russia

Abstract The high-temperature $\left(T=1880^{\circ} \mathrm{C}\right)$ Be-ions diffusion and electron irradiation influence on the AlN monocrystals optical properties were investigated. We demonstrated that Be diffusion into AlN leads to spectral properties change of the Raman scattering and infrared absorption. The analysis of the Raman and infrared absorption spectra of the AlN crystals, containing Be impurity, proves that Be impurity is a getter for intrinsic impurities, which are responsible for AlN crystals yellow color manifestation and spectral lines broadening. 